\title{
Combinatorial results on the Extended Star graph with Cross Connections
}

\author{
S. Pious Missier ${ }^{1}$, M. Evangeline Prathibha ${ }^{2}$, A. Anto Kinsley ${ }^{3}$ \\ ${ }^{I}$ Associate Professor, Department of Mathematics, V. O. Chidambaram College, Thoothukudi, India. \\ ${ }^{2}$ Research Scholar, Department of Mathematics, V.O. Chidambaram College, Thoothukudi, India \\ ${ }^{3}$ Associate Professor, Department of Mathematics, St. Xaviers's College (Autonomous), Palayamkottai, India.
}

Abstract: The extended star graph with cross-connections $\operatorname{ESC}(n, k)$ is a relatively new interconnection network topology, that has a hierarchical and recursive network that combines the versatility and robustness of star graph architectures. In this paper we have discussed some combinatorial results to find the number of nodes and edges of ESC $(n, k)$.

Keywords: Network, star graphs, extended star graphs.

\section{Introduction}

Many interconnection topologies have been proposed for highly parallel distributed-memory computers. The star graph is one of the interesting topologies studied due to its numerous attractive features including its regular structure. Efficient routing algorithms constitute one of the keys to the performance of a star graph system. The star graph has many advantages such as regularity, symmetry, low diameter, optimal fault tolerance. It offers a network with fewer interconnection edges and smaller communication delays compared to the hypercube. On the other hand, the star graph network is not modular and it lacks a basic building block. Hence a new interconnection network called the extended star graph has been proposed [1].

The extended star graph $(E S)$ is a scalable network which takes advantage of star graph and tree architectures [1]. The extended star graph exhibits improved diameter, cost factor and utilization factors. In addition, it has a low diameter, constant degree of connectivity and a basic building block.

In this paper we discuss some combinatorial results to find the number of nodes and edges of $\operatorname{ESC}(n, k)$.

\section{Extended star graph}

The extended star graph [1] is a new interconnection network which is modular and is a scalable network. The extended star graph architecture consists of two types of nodes, the processor elements (PEs) and the network controllers $(N C \mathrm{~s})$. The basic module of the extended star graph, represented by $E S(n, 1)$ consists of an $n$-star of $P E \mathrm{~s}, P_{1}, P_{2} \ldots . P_{n !}$ at level zero and one $N C$ represented as $P_{0}$ at level one. The $P E \mathrm{~s}$ of the $n$-star are connected to the $N C$ by links. The $E S(3,1)$ with two hierarchical levels is shown in Fig. 2.1. The PEs are all at the lowest (or zero) level and the $N C s$ are at higher levels. Using $n$ ! such building blocks we can construct an $E S(n, 2)$ with three levels of hierarchy. The number of hierarchical levels of $E S(n, k)$ is denoted as $n_{h}$ and its value is $k+1$.

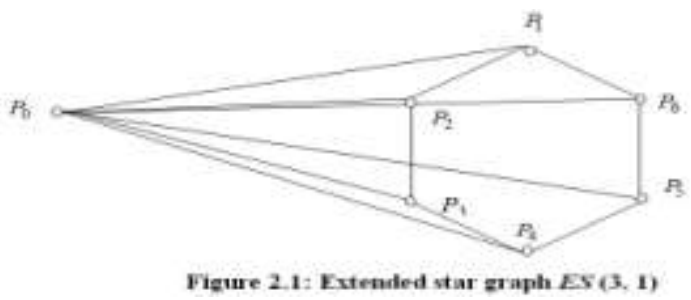

In general, we represent an extended star graph comprising of $n$ - stars and $(k+1)$ hierarchical levels by $E S(n, k)$. The ES $(n, k)$ can be built using $n ! E S(n, k-1) \mathrm{s}$. The PEs and NCs of the ES $(n, k)$ are represented by combination of permutations $P_{1}, P_{2} \ldots P_{n !}$ on $n$ symbols $1,2,3 \ldots n$. The combination of two permutations is denoted as $P_{i} P_{j}$, simply $P_{i j}$. Denote the set $\{1,2 \ldots n !\}$ as $\Lambda$. In the $E S(n, k)$, the $P E$ s are addressed by combination of $k$ permutations as $P_{i_{1} i_{2} \ldots i_{k}}$, where $i_{1}, i_{2}, \ldots i_{k} \in \Lambda$. In general, the nodes of an $n$-star at level $j$ are represented by combination of $(k-j)$ permutations, where $0 \leq j \leq k$. Thus a node of level $j$ is represented by $P_{i_{1} i_{2} \ldots i_{k-j}}$ and a node at level zero is represented by $P_{i_{1} i_{2} \ldots i_{k}}$ where $i_{1}, i_{2}, \ldots i_{k} \in \Lambda$. The symbol $P_{i_{1}}$ indicates 
the address of the top parent node at level $k-1, P_{i_{k-j-1}}$ corresponds to the parent node and $P_{i_{k-j}}$ represents the position of the node in the $n$-star. The addresses of all pairs of neighbouring PEs differ in the last component. Hence the $E S(n, k)$ is constructed by interconnecting $n$ ! number of $E S(n, k-1) \mathrm{s}$ and the $N C$ of level $k$ form an $E S(n, k)$. The $E S(2,1)$ and $E S(2,2)$ are shown in Fig. 2.2 (a) and (b).

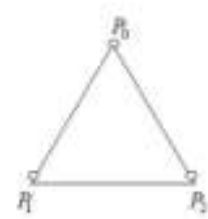

Figare 2.2(a) Fstended star zraph ES (2, 1)

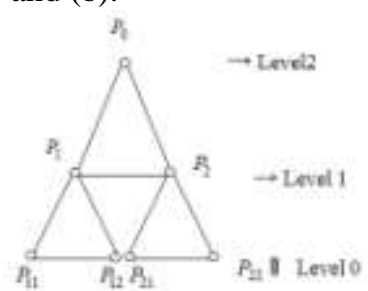

Figure 2.2(b): Extended star graph ES (2,2)

\section{Extended star graph with cross-connections}

In this section we propose an improvisation of the extended star graph $E S$, called the extended star graph with cross connections.

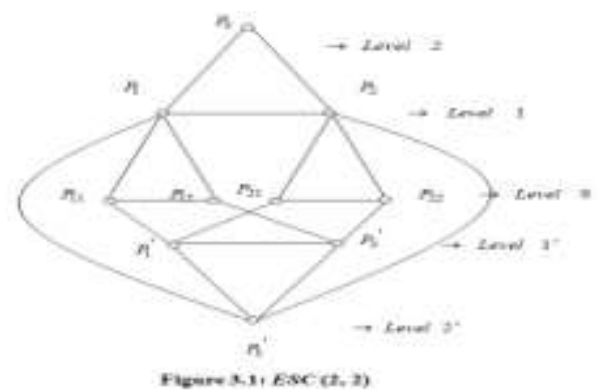

The extended star graph with cross-connections [1], ESC, is constructed as ES ( $n, k)$ and is built using basic building block, each basic building block represented by $\operatorname{ESC}(n, k)$ consists of n-star of processor elements $(P E \mathrm{~s})$, a network controller $(N C)$, links from each $P E$ to the $N C$ and additional links for interconnecting with other new basic building blocks as shown in Fig. 3.1.

There are cross -links forming a network among the $N C$ s and the $P E$ s. Also there are two $N C$ s with same address for all levels $j>0 ; P_{i_{1} i_{2}} \cdots i_{k-j}(N C)$ and $P_{i_{1} i_{2}}^{\prime} \cdots i_{k-j}\left(N C^{\prime}\right)$ where as each $P E$ of level zero represented by $P_{i_{1} i_{2}} \cdots i_{i_{k-1} i_{k}}$ is connected to two $N C$ s of level 1 and level $1^{\prime}$ with the addresses $P_{i_{1} i_{2}} \cdots i_{\mathrm{i}_{\mathrm{k}-1}}(N C)$ and $P_{i_{1} i_{2}}^{\prime} \cdots \cdots i_{k-1}\left(N C^{\prime}\right)$ respectively.

A node $N C$ at level $j(0<j<k)$ represented by $P_{i_{1} i_{2}} \cdots i_{k-j}$ is connected to two parent nodes $P_{i_{1} i_{2}} \cdots i_{\mathrm{i}_{k-j-1}}$ of level $j-1$ and $P_{i_{i} i_{2}}^{\prime} \cdots \mathrm{i}_{\mathrm{k}-\mathrm{j}-1}$ of level $(j-1)^{\prime}$. In addition an $N C$ at level $j, 0<j<k$ with address $P_{i_{i} i_{2}} \cdots \mathrm{i}_{\mathrm{k}-\mathrm{j}-1}$ is connected to $n$ ! child nodes at level $j+1$. An $\operatorname{ESC}(n, k)$ comprises of $(n !)^{\mathrm{k}-1} n$-stars of PEs at the lowest level of hierarchy and two $N C s$ at the highest level of hierarchy.

In general there are $2(n !)^{k-j-1} n$-stars of $N C$ s at level $j$. Hence there are $(n !)^{k-1}(n !)=(n !)^{k} P E$ s at the lowest level.

Hence there are three types of links in the $\operatorname{ESC}(n, k)$. They are (i) the star links connecting nodes of the $n$-stars, (ii) the $E S$ links forming a $n$ !- ary tree network with $N C$ s and $P E$ s and (iii) the cross links forming a network among the $N C$ 's and PEs. These links are shown in Fig. 3.2.

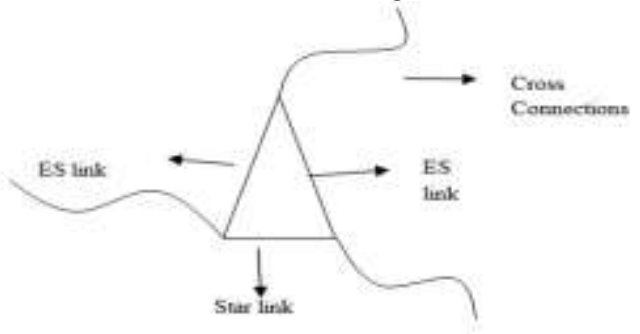

Figure 3.2, Basic building block of ES $(2, k)$ 


\section{Combinatorial Results}

We shall be using combinatorial techniques to determine the number of nodes and edges of $\operatorname{ESC}(n, k)$, keeping in mind its hierarchical structure. First, we shall quote some earlier results for our reference.

Theorem 3.1

The number of nodes of $E S(n, k)$ is given by $\sum_{j=0}^{k}(n !)^{j}$. [3]

\section{Theorem 3.2}

The number of nodes in any level $j$ of the extended star graph $E S(n, k)$ is $(n !)^{k-j}$.[3]

Theorem 3.3

The number of edges of $E S(n, k)$ is determined by the formula $\frac{(n+1) !}{2} \sum_{j=0}^{k-1}(n !)^{j} \cdot[3]$

\section{Theorem 3.4}

If $G$ is the graph $E S(n, k)$, then

(i) $\delta(G)=n$

(ii) $\quad \Delta(G)=n !+n \quad$ if $k \geq 2$

(iii) $\quad \Delta(G)=n$ ! if $k=1[3]$

Note: The following results have been derived combinatorially using the above mentioned results.

\section{Theorem 3.5}

The number of nodes of $\operatorname{ESC}(n, k)$ is given by $(n !)^{k}+2 \sum_{j=0}^{k-1}(n !)^{j}$

\section{Proof:}

It is known that the [3] number of nodes in an extended star graph is $\sum_{j=0}^{k}(n !)^{j}$ where $0 \leq j \leq k . \operatorname{In} \operatorname{ESC}(n, k)$, in addition to the nodes of $E S(n, k)$ we have the nodes of an $E S(n, k-1)$. Hence the number of nodes in $\operatorname{ESC}(n, k)$ is $\sum_{j=0}^{k}(n !)^{j}+\sum_{j=0}^{k-1}(n !)^{j}$. In other words, the number of nodes in $\operatorname{ESC}(n, k)$ is $(n !)^{k}+2 \sum_{j=0}^{k-1}(n !)^{j}$.

\section{Theorem 3.6}

The number of nodes in any level $j$, of an extended star graph with cross connections $\operatorname{ESC}(n, k)$ is $(n !)^{k-j}$ where $0 \leq j \leq k$ and the number of nodes in any level $1 \leq j^{\prime} \leq k$ is $(n !)^{k-j^{\prime}}$

\section{Proof:}

For all $k$ and all $n$, at level $k$ or $k^{\prime}$, the topmost hierarchical level, there is only one node, namely the NC $P_{0}$ or the NC $P_{0}^{\prime}$. In other words, there are $(n !)^{0}$ nodes at level $k$ and $k^{\prime}$ (i.e) $(n !)^{k-k}$ nodes at level $k$ (also in level $k^{\prime}$ ). At the subsequent level, namely level $k$-1(likewise for $k^{\prime}-1$ ), there is an $n$-star with $n$ ! nodes (i.e) $(n !)^{k-(k-1)}$ nodes. Each of these $n$ ! nodes in turn branch into $n$ ! nodes at level $k-2$ (and $k^{\prime}-2$ ) and so on and so forth. Hence we note that there are $(n !)^{2}$ (i.e) $(n !)^{k-(k-2)}$ nodes at level $k-2$ (similarly in $k^{\prime}-2$ ) and so on. Thus in general, at any level $j$ where $0 \leq j \leq k$, there are $(n !)^{k-j}$ nodes. Also there are $(n !)^{k-j^{\prime}}$ at any level $j^{\prime}$ where $1 \leq j^{\prime} \leq k$.

\section{Theorem 3.7}

The number of edges in $\operatorname{ESC}(n, k)$ is determined by the formula,

$$
\sum_{j=1}^{k}(n !)^{j}+\frac{(n+1) !}{2}\left\{\sum_{j=0}^{k-1}(n !)^{j}+\sum_{j=0}^{k-2}(n !)^{j}\right\}
$$

Proof: 
We know that $E S(n, k)$ has $\frac{(n+1) !}{2}\left\{\sum_{j=0}^{k-1}(n !)^{j}\right\}$ edges and that there are $\sum_{j=0}^{k-1}(n !)^{j} \operatorname{ES}(n, 1) \mathrm{s}[3]$. Since the $\operatorname{ESC}(n$, $k$ ) has an extra $\sum_{j=0}^{k-2}(n !)^{j} E S(n, 1)$ s we have $\frac{(n+1) !}{2}\left\{\sum_{j=0}^{k-2}(n !)^{j}\right\}$ edges. In addition to these edges, there are cross- connections connecting each node in level $j ; j=0,1,2 \ldots k$-1 to the nodes in level $i^{\prime} ; i=1,2 \ldots k$. There are $(n !)^{k}$ cross connections between level 0 and level $1^{\prime},(n !)^{k-1}$ cross connections between level 1 and level $2^{\prime}$ and so on. So there are $\sum_{j=1}^{k}(n !)^{j}$ cross connections in $\operatorname{ESC}(n, k)$ and this number also has

to be included in the total number of edges. Thus the total number of edges in $\operatorname{ESC}(n, k)$ is $\sum_{j=1}^{k}(n !)^{j}+\frac{(n+1) !}{2}\left\{\sum_{j=0}^{k-1}(n !)^{j}+\sum_{j=0}^{k-2}(n !)^{j}\right\}$.

\section{Theorem 3.8 (Recursive Equation)}

Let $p(G)$ and $q(G)$ denote the number of nodes and edges of a graph $G$ respectively. Then

(i) $\quad p(E S C(n, k))=n ! p(E S C(n, k-1))+2$.

(ii) $\quad q(\operatorname{ESC}(n, k))=n ! q(\operatorname{ESC}(n, k-1))+n !+(n+1)$ !

Proof:

$$
\text { (i) } \begin{aligned}
p(\operatorname{ESC}(n, k)) & =(n !)^{k}+2 \sum_{j=0}^{k-1}(n !)^{j} \\
& =(n !)^{k-1} \cdot(n !)+2+2\left\{n !+(n !)^{2}+\ldots+(n !)^{k-1}\right\} \\
& =n !\left\{(n !)^{k-1}+2 \sum_{j=0}^{k-2}(n !)^{j}\right\}+2 \\
& =n ! p(\operatorname{ESC}(n, k-1))+2 .
\end{aligned}
$$

(ii) $q(\operatorname{ESC}(n, k))=\sum_{j=1}^{k}(n !)^{j}+\frac{(n+1) !}{2}\left\{\sum_{j=0}^{k-1}(n !)^{j}+\sum_{j=0}^{k-2}(n !)^{j}\right\}$

which can be simplified to

$$
n !+(n+1)+n !\left\{\sum_{j=1}^{k-1}(n !)^{j}\right\}+\frac{(n+1) !}{2}\left\{n !+(n !)^{2}+\ldots+(n !)^{k-1}+n !+(n !)^{2}+\ldots+(n !)^{k-2}\right\}
$$

By further simplification we get

$$
n !+(n+1) !+n !\left\{\sum_{j=1}^{k-1}(n !)^{j}\right\}+\frac{(n+1) !}{2}\left\{\sum_{j=0}^{k-2}(n !)^{j}+\sum_{j=0}^{k-3}(n !)^{j}\right\}
$$

which equals $n !+(n+1) !+n !(q(E S C(n, k-1))$

Hence the result.

\section{Conclusion}

The above results help us to understand the structure of the $\operatorname{ESC}(n, k)$ and calculate the number of nodes and edges of the graph and also a recursive equation to find the same. These results help to calculate the number of nodes or edges of $\operatorname{ESC}(n, k)$ no matter, how large $n$ or $k$ may be and help to construct and visualize the network. Further, these results help in deriving the results involving other parameters of a network.

\section{References}

[1] A. Anto Kinsley, S. Somasundaram and C.Xavier, "Fault- tolerant Hierarchical Network of Star Graphs", Computer Science and Informatics, Vol. 29(1), March 1999,20-35.

[2] K.Day and A. Tripathi, "A Comparative Study of Topological Properties of Hypercubes and Star Graphs", IEEE Transactions on parallel and distribution systems Vol.5 (1), January 1994, 31-38.

[3] S. Pious Missier, M. Evangeline Prathibha and A. Anto Kinsley, April 2012, "Combinatorial results on the Extended Star Graph Topology ES (n, k)", Outreach, Vol. 5, 2011, 79-82. 